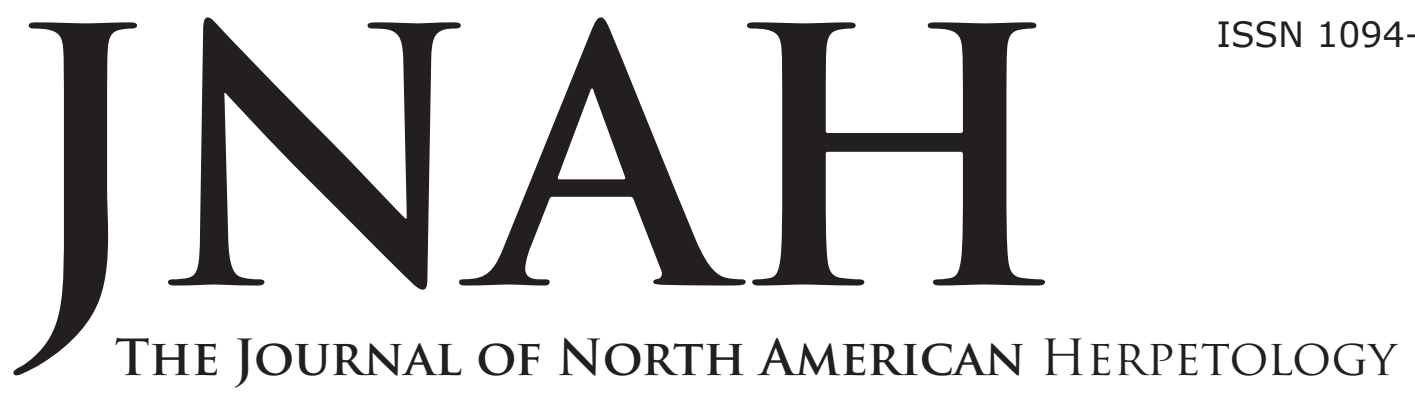

Volume 2016, Number 1

5 July 2016

jnah.cnah.org

\title{
COMPARING TWO TRANSECT METHODS FOR THE DETECTION OF RED-BACKED SALAMANDERS
}

\author{
JAY D. MCGHEE ${ }^{1,3}$ AND MICHAEL D. KILLIAN ${ }^{2}$ \\ ${ }^{1}$ Department of Natural Sciences, Northwest Missouri State University, \\ 800 University Drive, Maryville, Missouri 64468; E-mail: jmcghee@ \\ nwmissouri.edu. \\ ${ }^{2}$ Department of Biological Sciences, University of Mary Washington, 1301 \\ College Avenue, Fredericksburg, Virginia 22401; E-mail: mkillian@ \\ umw.edu. \\ ${ }^{3}$ Author for correspondence. jmcghee@nwmissouri.edu.
}

\begin{abstract}
Typical monitoring methods for terrestrial salamanders are subject to extensive variation, driven by the environmental conditions in effect during sampling. As rigorous salamander sampling methods are needed to monitor populations, there is a need to assess commonly used methods under a variety of environmental conditions. We hypothesized that of two methods used for capturing redbacked salamanders (Plethodon cinereus), leaf litter searches and natural cover searches, the latter would perform best for adults and in situations where moisture was limiting. We compared captures for paired transect surveys: one a leaf litter search and the other a natural cover search, relative to age, proximity to streams, rainfall events, vapor pressure deficit and season. We found that natural cover searches outperformed leaf litter searches when conducted away from streams and in the absence of rainfall. Natural cover searches performed better regardless of vapor pressure deficit and season (spring or fall). Natural cover searches detected more adults than juveniles. We recommend natural cover searches as more efficient than leaf litter searches.
\end{abstract}

Key Words: Monitoring, Sampling, Detection, Quadrat, Natural Cover, Leaf Litter

\section{INTRODUCTION}

Studies have been conducted over the past several years quantifying the effectiveness of detecting salamanders under a series of sampling methods (Hyde and Simons 2001, Marsh and Goicochea 2003, Williams and Berkson 2004, Richmond and Trombulak 2009). While these studies have found natural cover searches (searching cover objects in a constrained area) to be consistently efficient in regards to number of captures and cost and time-effective there is still uncertainty regarding how well these methods work under varying capture probabilities (Strain et al. 2009, Otto and Roloff 2011). Variables affecting the probability of capture for one method or another include species-specific behaviors, time of day, rainfall, temperature, adjacency to streams, and disturbance by logging (Hyde and Simons 2001, Williams and Berkson 2004, Hyde and Simons 2005). Salamander populations exist in a broad spectrum of habitats, where these aforementioned and other important conditions can be expected to differ. For example, in areas with high logging disturbance, low elevation, or limited stream ac- cess, there may be few salamanders in the population on the soil surface available for detection; consequently, knowledge of the efficiency of a particular method under such conditions will be important (Bailey et al. 2004a). There is a need, therefore, to continue to assess monitoring methods and to determine their relative efficacy as conditions change. As part of an extended salamander study in the Virginia Piedmont, we sought to assess two sampling methods used in terrestrial salamander monitoring: quadrat leaf litter searches and natural cover searches. We conducted our assessment by focusing on a single, common amphibian species: the red-backed salamander.

Salamander presence is commonly detected using a transect method (Jaeger 1994) which may include the use of quadrat leaf litter searches or natural cover searches. Quadrat sampling requires researchers to thoroughly search a set of samples of a fixed small area of leaf litter plus any ground cover present. Natural cover sampling involves searching, within a fixed area, for salamanders under the rocks and logs typically used as refuges by sal- 
amanders (Jaeger 1994, Jaeger and Inger 1994, Mitchell 2000). The method one chooses to determine the presence or absence of species, or to monitor populations is important if these methods differ substantially in efficacy, cost or man-hours in a given habitat. Generally, natural cover searches are understood to perform better for these criteria. Hyde and Simons (2001) found that natural cover searches produced less spatial and temporal variation in captures than quadrat leaf litter searches for most species, while Strain et al. (2009) found natural cover searches to have a higher capture rate while being more cost-effective in terms of number of species captured, and number of individuals captured. Otto and Roloff (2011), however, found that natural cover searches and quadrat leaf litter searches produced similar site occupancy rates for red-backed salamanders, though natural cover searches were significantly better for detecting changes in population occupancy over time. The higher performance of natural cover searches is generally attributed to the shorter sampling time required (Strain et al. 2009, Otto and Roloff 2011). If natural cover objects are limited at a site, however, or if natural cover objects are biased towards a specific size classes (Mathis 1990), then leaf litter quadrat searches may perform better for site-specific studies.

Red-backed salamander detectability depends on the proportion of the population available for capture on the surface, relative to the entire population, occurring both on the surface and beneath the soil (Bailey et al. 2004b). This species prefers cool, well-drained soils of neutral $\mathrm{pH}$, associated with sufficient leaf litter (Bogert 1952, Burger 1935, Wyman and Hawksley-Lescault 1987, Petranka 1998). Plethodontid salamanders respire through their skin rather than lungs, so must keep their skin moistened for gas exchange (Spotila 1972). Under dry conditions, a large proportion of the population may take refuge under the soil (Taub 1961, Heatwole 1962, Spotila 1972). The remaining smaller surface population may be more likely found under cover objects substantial enough to maintain the moisture level required by these animals or large enough for oviposition (Petranka 1998). Leaf litter being typically more extensive than the natural cover objects, may hold multiple individuals (i.e. juveniles and subordinate males) that cannot hold a territory associated with natural cover (Mathis 1990). Under certain conditions dominant males may move from their defended natural cover territories to hunt in the leaf litter, such as when food becomes limiting (i.e. low prey encounter rate) in their natural cover territory, necessitating active foraging, however this would be expected to occur only when the risk of desiccation was low (Jaeger 1980). Prey encounter rate and desiccation risk can be expected to vary seasonally and spatially, with both expected to be lowest in the early spring (when cool temperatures maintain high moisture levels) or in cooler, more humid riparian areas. Thus these might be the times and places when leaf litter sampling is preferred.

Over the course of a two-year salamander survey at C. F. Phelps Wildlife Management area in Northern Virginia, we conducted an assessment of leaf litter quadrat and natural cover searches under varying natural conditions. As natural cover objects hold more moisture relative to leaf litter, we hypothesized that natural cover searches (NC) would provide more adult red-backed salamander captures per sample under dry, warm conditions as opposed to cool, wet conditions. From this hypothesis, we predicted that average NC captures per transect would be higher than average leaf litter quadrat search (LLQ) captures per transect for 1 ) adult captures, 2) captures $>50 \mathrm{~m}$ from a stream, 3) captures in the absence of rain, 4) captures in autumn, and 5) captures in low humidity conditions. Further, we predicted that average LLQ captures per transect would be higher than average NC captures per transect for 6) juvenile captures, 7) captures $<50$ m from a stream, 8) captures during rain events, and 9) captures in spring.

\section{MATERIALS AND METHODS}

C. F. Phelps Wildlife Management Area (WMA) is located in Fauquier and Culpeper counties, Virginia bordering the eastern edge of the Rappahannock River in the Virginia Piedmont region. The WMA is 1837 hectares with elevations ranging between 200 and $400 \mathrm{~m}$ above sea level. Habitat includes deciduous hardwood forest, coniferous forest, areas of mixed hardwoods and conifers, and about 400 hectares of open fields leased for agriculture. The property holds several small streams that feed into the Rappahannock River.

Sampling locations were determined from randomly selected GPS coordinates on the property. At each sampling location we sampled along two transects (quadrat transect for leaf litter searches and natural cover transect for cover object searches) running parallel to each other $10 \mathrm{~m}$ apart. LLQ occurred within a $50 \mathrm{~m}$ transect laid along a randomly chosen azimuth. Five, $1-\mathrm{m}^{2}$ quadrats were placed at random within consecutive $10 \mathrm{~m}$ sections of the 50m transect (Jaeger 1994, Jaeger and Inger 1994, Mitchell 2000). Quadrats were searched by looking under all natural cover objects, such as rocks or logs, and by raking by hand through all leaf litter. NC occurred within a $3 \mathrm{~m}$ wide strip $10 \mathrm{~m}$ distant from the $50 \mathrm{~m}$ LLQ transect. All cover objects within this area were searched. Captured salamanders were identified to species and measured for snout-vent length and total length to determine age class (Petranka 1998). For each sample site (i.e. at the location of the two parallel transects), we measured air temperature and relative humidity. We used this information to calculate the vapor pressure deficit (VPD), i.e. the difference between the actual moisture in the air and the maximum amount of moisture the air could hold for a given temperature. We divided VPD into 2 categories: low VPD (air highly saturated with water) and high VPD (air with little water saturation) based on where a measurement fell in relation to the median VPD for all transects. We recorded the season (fall, spring, summer, winter) that sample was conducted within, the occurrence of rain, and whether the sample was located within $50 \mathrm{~m}$ of a stream (Hyde and Simons 2001). We used a

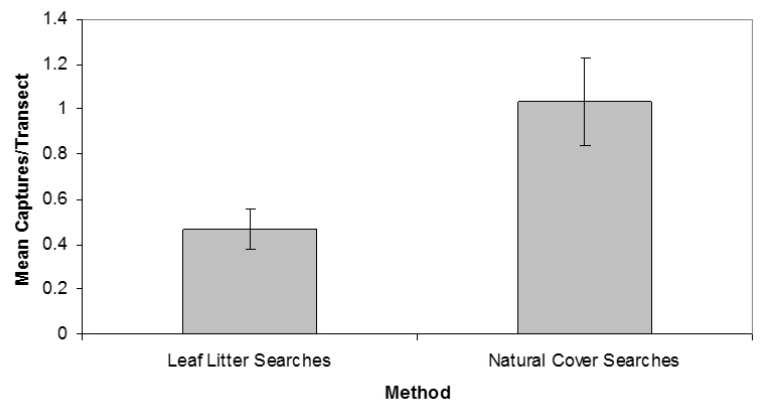

Figure 1. Mean ( \pm standard error) captures of red-backed salamanders per transect for two common monitoring methods: leaf litter quadrat searches and natural cover searches for $C$. F. Phelps WMA from April 2007 to April 2009 ( $n=90$ transects) 
series of paired t-tests $(a=0.05)$ to determine whether there was a difference between the average captures per transect for the LLQ and NC. To compare the relative variability between the two methods, we measured the coefficient of variation (CV), a standardized measure of variability calculated by dividing the standard deviation by the mean, for both NC and LLQ. We calculated the coefficient of determination $\left(r^{2}\right)$ between NC and LLQ across transect pairs to determine the level of consistency between capture methods. We also used a t-test to compare mean age class between LLQ and NC.

\section{RESULTS}

We sampled 90 pairs of transects, capturing 135 redbacked salamanders. We captured 42 using LLQ and 93 using NC. We captured $0.47 \pm 0.09$ (mean \pm standard error) individuals per transect using LLQ and $1.03 \pm 0.20$ individuals per transect using NC (Figure 1). NC produced more captures per transect than LLQ $(t=2.63$, $\mathrm{df}=178, P=0.009)$. Capture rate variability was high, but, similar for both methods (LLQ CV $=1.79$, NC CV $=$ 1.80). NC and LLQ were loosely correlated $\left(n=90, r^{2}=\right.$ $0.27, P<0.0001)$. Of red-backed salamanders captured, we found significantly more adults $\bar{x}_{\mathrm{Ad}}=2.23 \pm 0.26$ than juveniles $\bar{x}_{j u v}=1.68 \pm 0.18$ in NC searches $(t=1.75, d f$ $=45, P=0.043$ ), but no difference between adults $\bar{X}_{A d}$ $=1.21 \pm 0.11$ and juveniles $\overline{\mathrm{X}}_{\text {juv }}=1.26 \pm 0.10$ in LLQ searches $(\mathrm{t}=0.31, \mathrm{df}=31, P \stackrel{\mathrm{juv}}{=} 0.378)$. We were able to determine the distance to the nearest stream for 61 transect pairs. For transects within $50 \mathrm{~m}$ of a stream, we captured an average of $0.74 \pm 0.14$ individuals using LLQ and $1.13 \pm 0.24$ using NC, but these were not significantly different $(\mathrm{t}=1.68, \mathrm{df}=37, P=0.051)$. For transects $>50 \mathrm{~m}$ from a stream, we captured an average of 0.32 \pm 0.08 individuals using LLQ and $1.46 \pm 0.28$ using NC, which were different $(\mathrm{t}=2.97, \mathrm{df}=22, P=0.004)$. We examined 11 transect pairs that occurred during rain events, capturing an average of $0.45 \pm 0.21$ individuals using LLQ and $0.83 \pm 0.47$ using NC, but these were not significantly different $(\mathrm{t}=1.10, \mathrm{df}=10, P=0.148)$. In the absence of rain ( $n=78$ transect pairs), however, we captured $0.43 \pm 0.09$ individuals using LLQ, less than the $1.05 \pm 0.21$ using NC $(t=3.45, \mathrm{df}=77, P<0.001)$. NC produced more captures in both dry (high VPD, $\mathrm{n}=$ $45,1.04 \pm 0.31$ ) and saturated (low VPD, $n=46,1.02$ $\pm 0.24)$ conditions than LLQ $(0.39 \pm 0.12,0.53 \pm 0.12$; $\mathrm{t}_{\text {highvpd }}=2.40, \mathrm{df}=44, P=0.020, \mathrm{t}_{\text {lowvpd }}=2.38, \mathrm{df}=45$, $P \stackrel{\text { highvpd }}{=} 0.021$ ).

We used an ANOVA to determine whether seasons of different years could be pooled and found no significant differences in the number of captures between years for spring or autumn. We therefore pooled transect pairs by season across years and found that NC produced more captures on average in both autumn $(n=25,1.60 \pm$ $0.37)$ and spring $(n=32,1.42 \pm 0.43)$ than LLQ $(0.80 \pm$ $0.20,0.50 \pm 0.16 ; \mathrm{t}_{\text {fall }}=2.31, \mathrm{df}=24, P=0.030, \mathrm{t}_{\text {spring }}$ $=2.47, \mathrm{df}=31, P=0.019)$.

\section{DISCUSSION}

NC outperformed LLQ by producing more captures with similar variability. The correlation of our NC captures to our LLQ captures $\left(r^{2}=0.27\right)$ is comparable to Hyde and Simons (2001), who reported an $r^{2}=0.19$. While both NC and LLQ roughly measure the presence of aboveground salamanders, the low coefficient of determination means that other factors are driving most of the variation in captures per transect, a conclusion also supported by our high CVs. The level of variability we found in both meth- ods demonstrates that they are highly affected by multiple factors that impede their ability to produce reliable population estimates. These results are in agreement with previous assessments of monitoring methods (Hyde and Simons 2001, Williams and Berkson 2004).

NC seems to be biased towards adult, rather than juvenile, red-backed salamanders. A possible explanation for this finding is that the area below NC are likely held as territories (Jaeger 1981, Mathis 1990), and successful territory holders are more likely to be full-grown adults (Mathis 1990). However, Hyde and Simons (2001) tended to catch larger southern red-backed salamanders (Plethodon serratus) using LLQ, so this bias may not hold for all sites. In contrast to NC, our LLQ produced equal numbers of juveniles and adults, so it may be a preferred method if researchers desire to sample from multiple age classes. We suggest that juveniles are likely to be more common in leaf litter because they have failed to find and/or hold a territory, along with any subordinate adults in the area (Mathis 1990).

NC performed as well as LLQ near streams, but better than LLQ away from streams. Hyde and Simons (2001) found using multiple monitoring methods, that the southern red-backed salamander was more common in sites $>50 \mathrm{~m}$ from streams. If streams moderate temperature and humidity changes in the riparian areas surrounding them, more salamanders may be active in the leaf litter near streams because the risk of desiccation is reduced (Heatwole 1962). While this might imply LLQ may work best or at least as well as NC under higher moisture conditions, we found instead that NC performed better than LLQ regardless of the VPD. NC even performed as well as LLQ during rain events, though this may be because any particular rain event may not have been sufficient enough for a territory holder to leave a cover object, or because cover objects may be revisited frequently.

Related to this, we found that NC outperformed LLQ in the absence of rain events. While red-backed salamanders may be more active in the leaf litter during rain events, they retreat to cover objects or beneath the soil to avoid desiccation during dry periods (Heatwole 1962). Williams and Berkson (2004) found that red-backed salamanders were less likely to be detected under drier conditions, even under natural cover objects. We had hypothesized LLQ might perform better in spring because of increased moisture levels, but this was not supported by our data. Non-territory holding individuals may be less available for detection in the leaf litter for several reasons: they are too highly dispersed, there is not enough moisture, and the predation risk is too high to risk spending significant amounts of time on the soil surface.

NC appears to have greater reliability as a method for capturing salamanders compared to LLQ. As long as cover objects are available, some proportion of the redbacked salamander population will use them, apparently regardless of local water availability (VPD, proximity to streams, rain events) or season. We recommend the use of NC over LLQ when sampling for red-backed salamanders. We stress, however, that our study provides no information on the functional relationship between the detection probability associated with NC and the true population size. Previous studies have suggested NC carries a high, but variable detection probability (Williams and Berkson 2004). This implies that the relationship between NC detection probability and true population size will be subject to high uncertainty, and possibly non-linear, and may need to be estimated on a site-specific ba- 
sis.

\section{ACKNOWLEDGMENTS}

We thank J. Ferdinandsen, manager of C. F. Phelps WMA, as well as R. Hughes \& J. Sims of Virginia Game and Inland Fisheries for their helpful cooperation and support. We also thank University of Mary Washington students Carly Byers, Sarah Almahdali, Jennifer Clary, Hillary Adams, and Ramsey Hanna for their assistance in the field. We thank the students of J. D. McGhee's animal ecology classes for Fall 2007 and 2008 for their help in field sampling.

\section{LITERATURE CITED}

Bailey, L. L., T. R. Simons, and K. H. Pollock. 2004a. Estimating detection probability parameters for plethodon salamanders using the robust capture-recapture design. Journal of Wildlife Management 68:1 - 13 .

Bailey, L. L., T. R. Simons, and K. H. Pollock. 2004b. Spatial and temporal variation in detection probability of plethodon salamanders using the robust capture-recapture design. Journal of Wildlife Management 68:14 - 24.

Bogert, C. M. 1952. Relative abundance, habitats, and normal thermal levels of some Virginia salamanders. Ecology 33: $16-30$.

Burger, J.W. 1935. Plethodon cinereus (Green) in eastern Pennsylvania and New Jersey. The American Naturalist 64:578 - 586 .

Heatwole, H. 1962. Environmental factors influencing local distribution and activity of the salamander, Plethodon cinereus. Ecology 43:460 - 472

Hyde, E.J., and T.R. Simons. 2001. Sampling plethodontid salamanders: sources of variability. Journal of Wildlife Management 65:624-632.

Hyde, E. J., and T. R. Simons. 2005. Monitoring salamander populations in Great Smoky Mountains National Park Pages 300 - 306 in M. Lannoo, editor. Amphibian declines: The conservation status of United States species. University of California Press, Berkeley, California.

Jaeger, R. G. 1980. Microhabitats of a terrestrial forest salamander. Copeia 1980: 265 - 268.

Jaeger, R. G. 1981. Dear enemy recognition and the costs of aggression between salamanders. The American Naturalist 117: 962 - 974.

Jaeger, R. G. 1994. Transect sampling. Pages 103-106 in: Measuring and monitoring biological diversity: standard methods for amphibians. W. R. Heyer, M. A. Donnelly, R. W. McDiarmid, L. C. Hayek, and M. S.
Foster, (eds.). Smithsonian Institution Press, Washington, D. C.

Jaeger, R. G. and R. F. Inger. 1994. Quadrat sampling. Pages 97-102 in: Measuring and monitoring biological diversity: standard methods for amphibians. W. R. Heyer, M. A. Donnelly, R. W. McDiarmid, L. C. Hayek, and M. S. Foster, (eds.). Smithsonian Institution Press, Washington, D. C.

Marsh, D. M. and M. A. Goicochea. 2003. Monitoring terrestrial salamanders: biases caused by intense sampling and choice of cover objects. Journal of Herpetology 37: $460-466$.

Mathis, A. 1990. Territoriality in a terrestrial salamander: the influence of resource quality and body size. Behavior 112: 162 - 175 .

Mitchell, J. C. 2000. Amphibian monitoring methods \& field guide. Smithsonian National Zoological Park Conservation and Research Center, Front Royal, Virginia.

Otto, C. R. V. and G. J. Roloff. 2011. Comparing cover object and leaf litter surveys for detecting red-backed salamanders, Plethodon cinereus. Journal of Herpetology 45: $256-260$.

Petranka, J. W. 1998. Salamanders of the United States and Canada. Smithsonian Institution Press, Washington, D. C.

Richmond, L. S. and S. C. Trobulak. 2009. Distribution of red-backed salamander (Plethodon cinereus) with respect to cover-object characteristics in the Green Mountains of Vermont. Northeastern Naturalist $16: 13-26$

Spotila, J. R. 1972. Role of temperature and water in the ecology of lungless salamanders. Ecological Monographs 42:95-125.

Strain, G. F., R. L. Raesly, and R. H. Hildebrand. 2009. A comparison of techniques to sample salamander assemblages along highland streams of Maryland. Environmental Monitoring and Assessment 156: 1 $-16$.

Taub, F. B. 1961. The distribution of the red-backed salamander, Plethodon c. cinereus, within the soil. Ecology $42: 681-698$.

Williams, A. K., and J. M. Berkson. 2004. Reducing false absences in survey data: detection probabilities of red-backed salamanders. Journal of Wildlife Management 68:418-428.

Wyman, R. L. and D. S. Hawksley-Lescault. 1987. Soil acidity affects distribution, behavior, and physiology of the salamander Plethodon cinereus. Ecology $68: 1819-1827$. 